\title{
Beam size and position measurement based on logarithm processing algorithm in HLS II ${ }^{*}$
}

\author{
CHENG Chao-Cai (() $)^{1)}$ SUN Bao-Gen( $)^{2)} \quad$ YANG Yong-Liang() ZHOU Ze-Ran() \\ LU Ping() WU Fang-Fang() WANG Ji-Gang() TANG Kai() \\ LUO Qing() LI Hao() ZHENG Jia-Jun() DUAN Qing-Ming() \\ National Synchrotron Radiation Laboratory, University of Science and Technology of China, Hefei, 230029, China
}

\begin{abstract}
A logarithm processing algorithm to measure beam transverse size and position is proposed and preliminary experimental results in Hefei Light Source II (HLS II) are given. The algorithm is based on only 4 successive channels of 16 anode channels of multianode photomultiplier tube (MAPMT) R5900U-00-L16 which has typical rise time of $0.6 \mathrm{~ns}$ and effective area of $0.8 \times 16 \mathrm{~mm}$ for a single anode channel. In the paper, we firstly elaborate the simulation results of the algorithm with and without channel inconsistency. Then we calibrate the channel inconsistency and verify the algorithm using general current signal processor Libera Photon in low-speed scheme. Finally we get turn-by-turn beam size and position and calculate the vertical tune in high-speed scheme. The experimental results show that measured values fit well with simulation results after channel differences are calibrated and the fractional part of the tune in vertical direction is 0.3628 which is very close to the nominal value 0.3621 .
\end{abstract}

Key words: logarithm processing algorithm, beam size and position, multianode photomultiplier tube, channel inconsistency

PACS: 29.20.db, 29.85.Ca, 29.90.+r

\section{Introduction}

In electron storage ring and collider complex, beam transverse profile measurement based on visible light from synchrotron radiation (SR) is widely used [1]. In general, CCD can be applied to measure beam size and position steadily and online. But conventional CCD has a long exposure time and very low frame rate. In HLS II, there has been installed a CCD camera to measure beam profile and monitor the machine status. The CCD camera has the maximum frame rate of $205 \mathrm{~Hz}$ at the full resolution $(640 \times 480)$, and the electron beam go through about $22 \mathrm{k}$ turns within single frame interval. As a result, the measurement results are indeed the average of multiple bunches in multiple turns.

In order to track the beam size and position of single bunch, it is necessary to acquire transient light intensity information utilizing high speed photodetector and data acquisition system to ensure that the transformed light pulse are not piled up along the pulse train. The typical schemes are VEPP-4M [2] and CESR-c [3] which calculate the turn-by-turn or bunch-by-bunch beam size and position to study beam-beam interaction, beam dynam- ics, etc. In these measurement schemes, dozens of channel information up to 16 or 32 are sampled in parallel to fit the shape of Gaussian beam spot, which increase the costs and complexity. Therefore, we put forward a logarithm processing algorithm to calculate the beam size and position which needs only 4 successive channels, and carry out some experiments to verify the algorithm.

\section{Principle of the measurement system}

At the moment there are 4 applications in the visible diagnostic beam line at $15^{\circ}$ outlet of bending magnet in HLS II. Fig. 1 shows the block diagram of the measurement system for CCD and vertical MAPMT. Synchrotron radiation is first reflected by water-cooled Oxygen Free Copper (OFC) mirror in vacuum chamber and then the visible light pass through several mirrors and a main focusing lens with magnification of 1 before reaching the optical table. MAPMT and CCD [4] share the same magnification of 2 by front-end optical path. Then the beam spot reaches the horizontal and vertical MAPMT cathode with magnification of 6 and magnification of 24 respectively. Magnification can be

Received 31 Jul 2015

* Supported by National Natural Science Foundation of China $(11005105,11175173)$

1) E-mail: ccc1989@mail.ustc.ac.cn

2) E-mail: bgsun@ustc.edu.cn

() 2013 Chinese Physical Society and the Institute of High Energy Physics of the Chinese Academy of Sciences and the Institute of Modern Physics of the Chinese Academy of Sciences and IOP Publishing Ltd 
altered to meet the need in different machine status. The two MAPMTs are fixed on three-dimensional translation stage so as to find the image plane conveniently. Front-end circular slit constraints the opening angle of SR within $\pm 3.8 \mathrm{mrad}$ to decrease the depth of field error and diffraction error 5]. A band pass filter with $500 \mathrm{~nm}$ peak wavelength and $10 \mathrm{~nm}$ full width at half maximum (FWHM) is used to allow quasi-monochromatic light to get to the sensitive area of MAPMT.

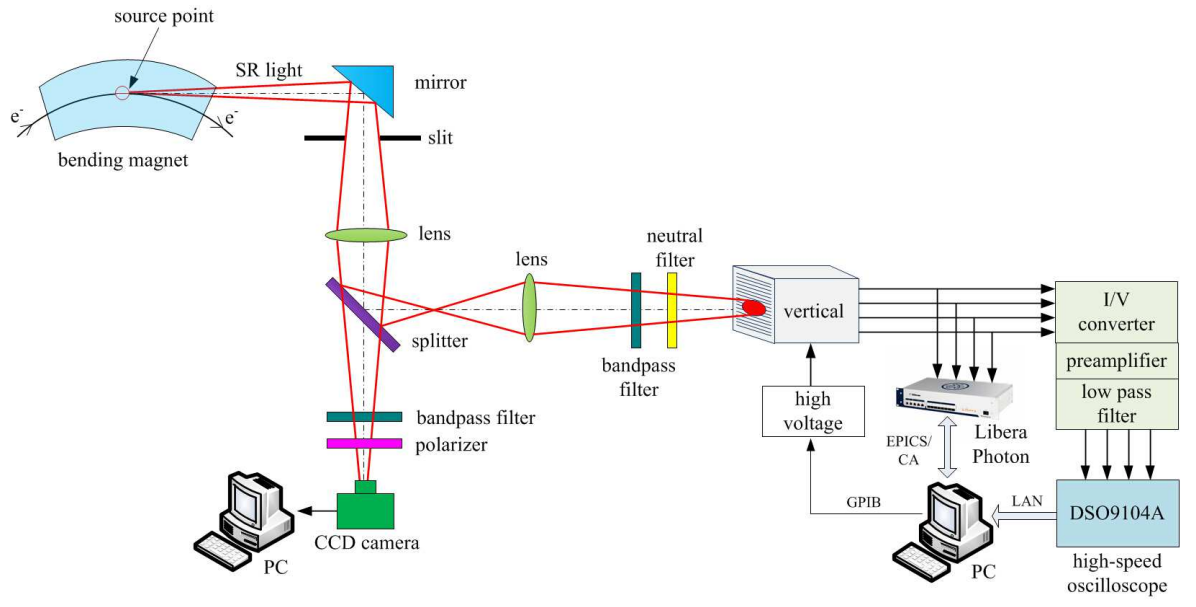

Fig. 1. Block diagram of the measurement system.

The measurement system works in two schemes. In low-speed scheme, photocurrent of 4 successive channels is sampled by general current signal processor Libera Photon from Instrumentation Technologies and then sent to remote PC by EPICS/CA protocol, where we can calibrate the channel inconsistency, implement the logarithm processing algorithm to extract beam size and position, and control the negative high voltage to protect the MAPMT. In high-speed scheme, photocurrent is firstly conditioned by analog front-end electronics (AFE) including current to voltage conversion, preamplifier and low pass filter. Then transformed signals are sampled simultaneously by high speed oscilloscope DSO9104A and acquired over Local Area Network (LAN) by remote PC where we can calculate turn-by-turn beam size and position, perform Fast Fourier Transformation (FFT), etc.

\section{Logarithm processing algorithm [6]}

As SR light irradiated by electron beam from bending magnet in storage ring presents Gaussian distribution, we assume SR light intensity distribution function $\Phi(x)$ as Eq. (1).

$$
\Phi(x)=\Phi_{0} \exp \left[-\left(\frac{x-\delta}{\sqrt{2} \sigma}\right)^{2}\right] .
$$

where $\Phi_{0}$ is the maximum light intensity; $\delta$ is beam displacement that center of the beam spot offsets specified center of the MAPMT; $\sigma$ is beam size.

Based on the assumption of Gaussian distribution, the SR light intensity distribution at MAPMT is shown in Fig. 22 The rectangular region of $I_{1}, I_{2}, I_{3}$ and $I_{4}$ represents effective photosensitive areas with $0.2 \mathrm{~mm}$ gap between them.

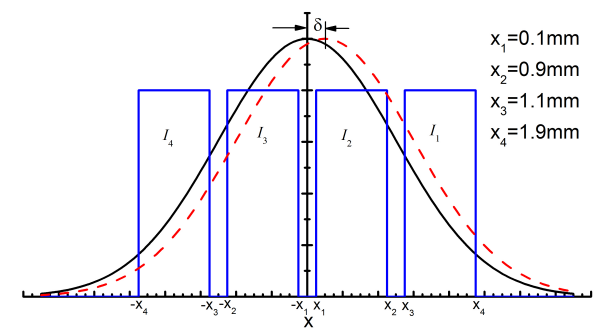

Fig. 2. Light intensity distribution at MAPMT.

Photocurrent of $I_{1}, I_{2}, I_{3}$ and $I_{4}$, which derives from 4 successive channels, is proportional to the integral of light intensity and can be expressed by the subtraction of two error functions shown in Eq. (2).

$$
\left\{\begin{array}{l}
I_{1}=\int_{x_{3}}^{x_{4}} \Phi(x) \mathrm{d} x=I_{0}\left[\operatorname{erf}\left(\frac{x_{4}-\delta}{\sqrt{2}}\right)-\operatorname{erf}\left(\frac{x_{3}-\delta}{\sqrt{2}}\right)\right] \\
I_{2}=\int_{x_{2}}^{x_{2}} \Phi(x) \mathrm{d} x=I_{0}\left[\operatorname{erf}\left(\frac{x_{2}-\delta}{\sqrt{2}}\right)-\operatorname{erf}\left(\frac{x_{1}-\delta}{\sqrt{2}}\right)\right] \\
I_{3}=\int_{-x_{2}}^{x_{1}} \Phi(x) \mathrm{d} x=I_{0}\left[\operatorname{erf}\left(\frac{-x_{1}-\delta}{\sqrt{2}}\right)-\operatorname{erf}\left(\frac{-x_{2}-\delta}{\sqrt{2}}\right)\right] \\
I_{4}=\int_{-x_{4}}^{-x_{3}} \Phi(x) \mathrm{d} x=I_{0}\left[\operatorname{erf}\left(\frac{-x_{3}-\delta}{\sqrt{2}}\right)-\operatorname{erf}\left(\frac{-x_{4}-\delta}{\sqrt{2}}\right)\right] .
\end{array}\right.
$$

where $\operatorname{erf}(x)=\frac{2}{\sqrt{\pi}} \int_{0}^{x} \mathrm{e}^{-t^{2}} \mathrm{~d} t ; x_{1}=0.1 \mathrm{~mm}, x_{2}=0.9 \mathrm{~mm}$, $x_{3}=1.1 \mathrm{~mm}$ and $x_{4}=1.9 \mathrm{~mm} ; I_{0}$ is the maximum anode photocurrent. 


\subsection{Ideal case}

In ideal condition when the 4 continuous channels have the same response characteristic, we define ideal size signal $S_{\ln }(\sigma, \delta)$ and ideal position signal $P_{\ln }(\sigma, \delta)$ in Eq. (3).

$$
\left\{\begin{array}{l}
S_{\ln }(\sigma, \delta)=\left[\ln \left(I_{2} I_{3} / I_{1} I_{4}\right)\right]^{-1 / 2} \\
P_{\ln }(\sigma, \delta)=\frac{\ln \left(I_{1} I_{2} / I_{3} I_{4}\right)}{\ln \left(I_{2} I_{3} / I_{1} I_{4}\right)}
\end{array}\right.
$$

Substituting the integral result shown in Eq. (2) into Eq. (3) and simplifying it, we can obtain how the size and position signal vary from size and position illustrated in Fig. 3. When beam size increases from $0.2 \mathrm{~mm}$ to $2 \mathrm{~mm}$ with $0 \mathrm{~mm}$ beam displacement, size signal $S_{\ln }(\sigma, \delta)$ has a good linear relation with size $\sigma$ and the linear fitting equation can be expressed by $S_{\ln }(\sigma)=0.03924+0.69 \sigma$. When beam size varies within $0.8 \mathrm{~mm} \sim 2 \mathrm{~mm}$, beam displacement $\delta$ has an effect on normalized size signal within $1 \%$. Meanwhile, position signal $P_{\ln }(\sigma, \delta)$ has a good linear relation with beam displacement when beam displacement varies from $-2 \mathrm{~mm}$ to $2 \mathrm{~mm}$ and linear fitting equation is $P_{\ln }(\delta)=2 \delta$. Furthermore, beam size has an impact on position signal sensitivity within $1 \%$.
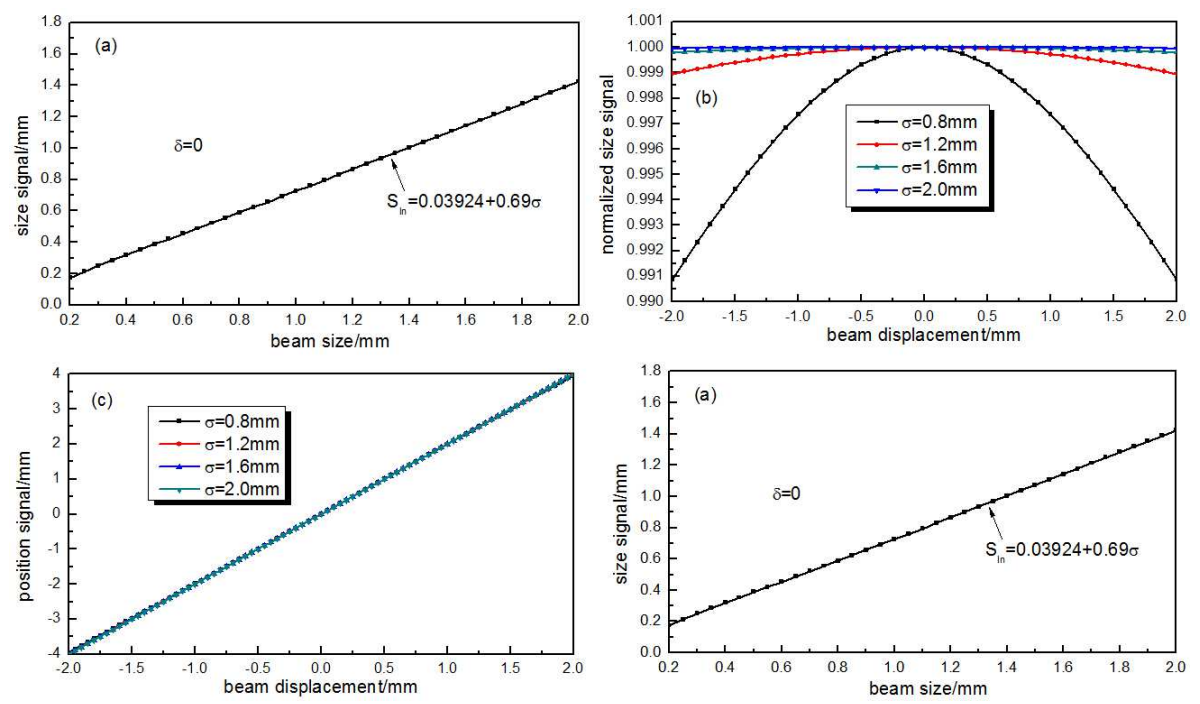

Fig. 3. The simulation results of ideal beam size and position signal in different conditions: (a) size signal versus beam size when $\delta=0 \mathrm{~mm}$; (b) normalized size signal versus beam displacement when $\sigma=0.8 \sim 2.0 \mathrm{~mm}$; (c) position signal versus beam displacement when $\sigma=0.8 \sim 2.0 \mathrm{~mm}$; (d) position signal sensitivity versus beam size.

From the above simulation results, we can get the approximate equations expressed in Eq. (4).

$$
\left\{\begin{array}{l}
S_{\ln }(\sigma, \delta) \approx S_{\ln }(\sigma)=0.03924+0.69 \sigma \\
P_{\ln }(\sigma, \delta) \approx P_{\ln }(\sigma)=2 \delta
\end{array}\right.
$$

\subsection{Non-ideal case}

Given the channel inconsistency resulting from anode uniformity and cross-talk among channels, we introduce the channel gain factor $g_{i}(i=1,2,3,4)$ to correct channel inconsistency based on linear approximation, as is shown in Eq. (5).

$$
\left\{\begin{aligned}
\hat{S}_{\ln }(\sigma, \delta) & =\left[\ln \left(\hat{I}_{2} \hat{I}_{3} / \hat{I}_{1} \hat{I}_{4}\right)\right]^{-1 / 2} \\
& =\left[\ln \left(g_{2} g_{3} / g_{1} g_{4}\right)+\ln \left(I_{2} I_{3} / I_{1} I_{4}\right)\right]^{-1 / 2} \\
\hat{P}_{\ln }(\sigma, \delta) & =\frac{\ln \left(\hat{I}_{1} \hat{I}_{2} / \hat{I}_{3} \hat{I}_{4}\right)}{\ln \left(\hat{I}_{2} \hat{I}_{3} / \hat{I}_{1} \hat{I}_{4}\right)} \\
& =\frac{\ln \left(g_{1} g_{2} / g_{3} g_{4}\right)+\ln \left(I_{1} I_{2} / I_{3} I_{4}\right)}{\ln \left(g_{2} g_{3} / g_{1} g_{4}\right)+\ln \left(I_{2} I_{3} / I_{1} I_{4}\right)} .
\end{aligned}\right.
$$

where $\hat{S}_{\ln }(\sigma, \delta)$ is actual size signal, $\hat{P}_{\ln }(\sigma, \delta)$ is actual position signal, $\hat{I}_{i}=g_{i} I_{i}(i=1,2,3,4)$ represents actual anode photocurrent from 4 successive channels with channel inconsistency.

If we define $d_{s}=\ln \left(g_{2} g_{3} / g_{1} g_{4}\right), d_{p}=\ln \left(g_{1} g_{2} / g_{3} g_{4}\right)$ and put Eq. (4) into Eq. (5), we can get:

$$
\left\{\begin{aligned}
\hat{S}_{\ln }(\sigma, \delta) \approx & S_{\ln }(\sigma)\left[1+\tilde{d}_{s} S_{\ln }^{2}(\sigma)\right]^{-1 / 2} \\
\hat{P}_{\ln }(\sigma, \delta) \approx & P_{\ln }(\delta)\left[1+\tilde{d}_{s} S_{\ln }^{2}(\sigma)\right]^{-1}+ \\
& \tilde{d}_{p} S_{\ln }^{2}(\sigma)\left[1+\tilde{d}_{s} S_{\ln }^{2}(\sigma)\right]^{-1}
\end{aligned}\right.
$$

where $\tilde{d}_{s}$ and $\tilde{d}_{p}$ are gain correction factors.

According to Eq. (6), we know that actual position signal has a good linear relation with beam position, but actual size signal presents a huge nonlinearity with size. In addition, $\tilde{d}_{s}$ and $\tilde{d}_{p}$ can be induced by slope and intercept of $\hat{P}_{\ln }(\sigma, \delta)$ if beam size and magnification can be acquired in advance. Hence, modified size signal $\tilde{S}_{\ln }(\sigma, \delta)$ and modified position signal $\tilde{P}_{\ln }(\sigma, \delta)$ can be expressed in 
Eq. (7).

$$
\left\{\begin{array}{l}
\tilde{S}_{\ln }(\sigma, \delta)=\left[\ln \left(\hat{I}_{2} \hat{I}_{3} / \hat{I}_{1} \hat{I}_{4}\right)-\tilde{d}_{s}\right]^{-1 / 2} \\
\tilde{P}_{\ln }(\sigma, \delta)=\frac{\ln \left(\hat{I}_{1} \hat{I}_{2} / \hat{I}_{3} \hat{I}_{4}\right)-\tilde{d}_{p}}{\ln \left(\hat{I}_{2} \hat{I}_{3} / \hat{I}_{1} \hat{I}_{4}\right)-\tilde{d}_{s}} .
\end{array}\right.
$$

\section{Experimental results}

\subsection{Low-speed scheme}

In low-speed scheme, the horizontal measurement results are described. In order to simulate the movement of beam center, we move the three-dimensional translation stage for horizontal MAPMT horizontally. The movement range is from $13.0 \mathrm{~mm}$ to $16.8 \mathrm{~mm}$ with 0.1 $\mathrm{mm}$ step. The process of movement is very short, so the beam size measured by CCD remains unchanged.

Figure 4 shows how the average anode output current varies with the moved position. $\mathrm{CH} 4, \mathrm{CH} 5, \mathrm{CH} 6$ and $\mathrm{CH} 7$ are corresponding to channel number 4, 5, 6 and 7 respectively. When the horizontal translation stage increases, average anode current of $\mathrm{CH} 4$ and $\mathrm{CH} 5$ increases and it is just opposite for $\mathrm{CH} 6$ and $\mathrm{CH} 7$, which roughly indicates the beam spot center is moving from $\mathrm{CH} 7$ to CH4. In the middle point of $14.9 \mathrm{~mm}$, anode current of $\mathrm{CH} 4$ and $\mathrm{CH} 7$ are approximate the same, and so does it for $\mathrm{CH} 5$ and $\mathrm{CH} 6$. Thus this position can be thought to be the absolute center.

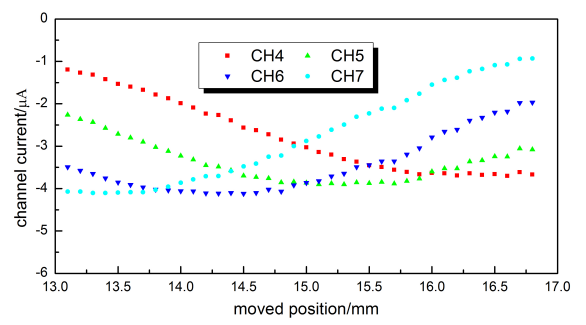

Fig. 4. Average anode current of 4 successive channels.

Putting average anode current into Eq. (5), we can get how the actual position signal varies with the simulated beam displacement shown in Fig. 5. The horizontal coordinate axis has been adjusted by subtracting the absolute center. Applying linear fitting to the raw discrete points, we can get the fitting equation with slope of 1.7894 and intercept of -0.1206 . Actual position signal has a linear relation with simulated beam displacement as described in Eq. (6). However, the fitting curve does not cross the point $(0,0)$, and slope does not equal to the simulation result in ideal case shown in Fig. 3(c). The difference mainly comes from the channel inconsistency and must be calibrated.

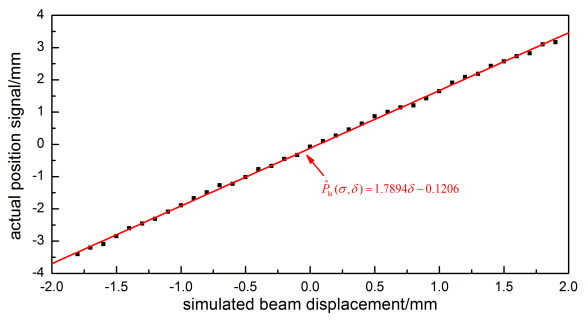

Fig. 5. Actual position signal with channel inconsistency.

Substituting the magnifying beam size imaged at the horizontal MAPMT cathode into Eq. (6), we can get the measured gain correction factors $\tilde{d}_{s}=0.0590$ and $\tilde{d}_{p}=-$ 0.0675 on the basis of the fitting equation shown in Fig. 5 , As a consequence, the relationship between the modified position and simulated position can be illustrated in Fig. 6. Linear fitting results show that the modified position signal has a good linear relation with simulated beam displacement and the fitting curve cross point $(0,0)$ with slope of 2.002 and intercept of -0.008 which agrees well with the simulation result in Fig .3(c).

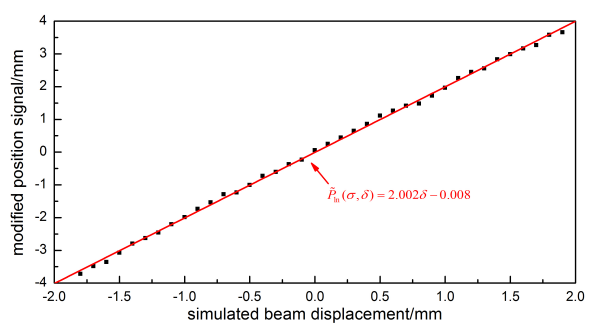

Fig. 6. Modified position signal.

Beam size calculated by direct equation in Eq. (5) and modified equation in Eq. (7) is illustrated in Fig. 7 respectively. Direct and modified beam size gradually decreases when beam center is moved from $-1.8 \mathrm{~mm}$ to $1.9 \mathrm{~mm}$ although the modified beam size signal which is proportional to beam size should remain almost unchanged according to Fig .3(b). But the modified beam size is more close to the nominal value $0.332 \mathrm{~mm}$ measured by CCD and is more reliable.

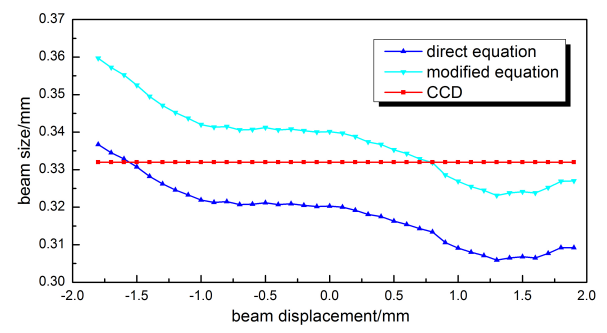

Fig. 7. Direct and modified beam size.

\subsection{High-speed scheme}

In high-speed scheme, we take the vertical measurement results for example. Single bunch fills in the storage ring with $3 \mathrm{~mA}$ beam current and electron beam is 
excited by swept frequency signal which comes from the tracking generator module in the spectrum analyzer. Signals from AFE are continuously sampled 500,000 points with $10 \mathrm{GS} / \mathrm{s}$ sample rate for 4 channels. External trigger signal with frequency of $4.534 \mathrm{MHz}$ synchronized with the revolution frequency is applied to keep pace with the pulse signal produced by SR light. Fig. 8 shows the peak values of each turn extracted from raw data points with peak search algorithm. As the sample points per one period is about 2200 , we can sample 227 turns data in total. $\mathrm{CH} 8, \mathrm{CH} 9, \mathrm{CH} 10$ and $\mathrm{CH} 11$ are corresponding to channel number 8, 9, 10 and 11 respectively. Peak value of $\mathrm{CH} 9$ and $\mathrm{CH} 10$ is larger than $\mathrm{CH} 8$ and $\mathrm{CH} 11$ indicating that beam center is located between the $\mathrm{CH} 9$ and CH10 which satisfies the conditions of the algorithm.

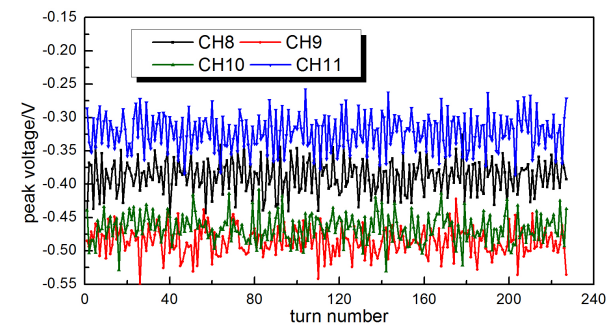

Fig. 8. Peak values of each turn for 4 successive channels.

Applying FFT to all the 500,000 points raw data and enlarging the view of spectrum of CH9 in frequency range of $0 \mathrm{~Hz}$ to $10 \mathrm{MHz}$ shown in Fig. 9, we can clearly see vertical synchronous oscillation sidebands appear at frequency of $1.620 \mathrm{MHz}, 2.900 \mathrm{MHz}, 6.160 \mathrm{MHz}$ and $7.440 \mathrm{MHz}$ except for two main harmonic components at the frequency of $4.530 \mathrm{MHz}$ and $9.060 \mathrm{MHz}$ which are roughly equal to the integral multiple of revolution frequency. These sideband elements result from the interactions between the synchronous light and slit. In HLS II, frequency at $1.620 \mathrm{MHz}$ and $6.160 \mathrm{MHz}$ correspond to betatron oscillation in vertical direction, and frequency at $2.900 \mathrm{MHz}$ and $7.440 \mathrm{MHz}$ are the mirrored components of vertical betatron oscillation.

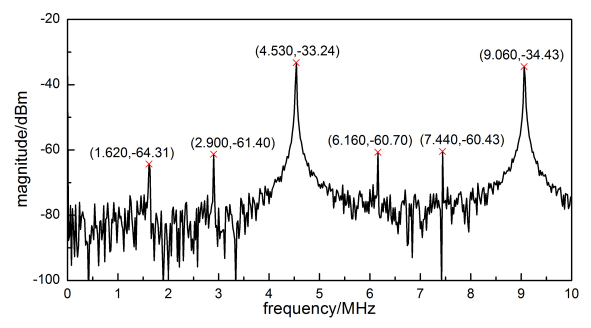

Fig. 9. Spectrum of CH9.

With the channel inconsistency being calibrated and logarithm processing algorithm being adopted with 227 turns peak values, we can get the turn-by-turn beam size and position shown in Fig. 10. From Fig. 10(a), beam size varies between $64.5 \mu \mathrm{m}$ and $93.5 \mu \mathrm{m}$ with the average of $76.4 \mu \mathrm{m}$. From Fig. 10(b), beam position varies between $-8.2 \mu \mathrm{m}$ and $22.6 \mu \mathrm{m}$ and the average value is $8.0 \mu \mathrm{m}$. Meanwhile, we record the beam size with CCD which tells that the beam size is about $70 \mu \mathrm{m}$. Our average result of beam size is larger than the beam size from CCD for about $8 \%$ which may result from the amplification of light path.
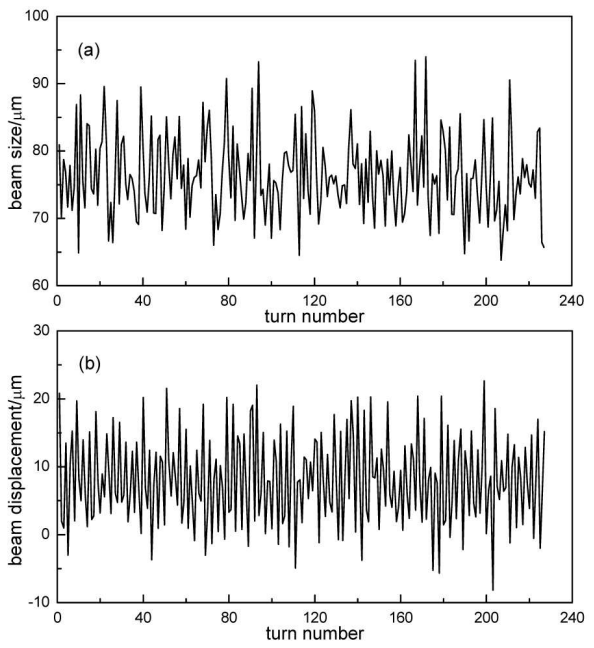

Fig. 10. Beam size and position for 227 turns: (a) beam size; (b) beam position.

Applying FFT to the turn-by-turn beam position and getting rid of the dc component, we can obviously see a peak signal at the frequency of $1.645 \mathrm{MHz}$ corresponding to the vertical betatron oscillation sideband shown in Fig. 11. So the vertical fractional tune is 0.3628 with $4.534 \mathrm{MHz}$ revolution frequency. The measured result is in good agreement with the nominal value 0.3621.

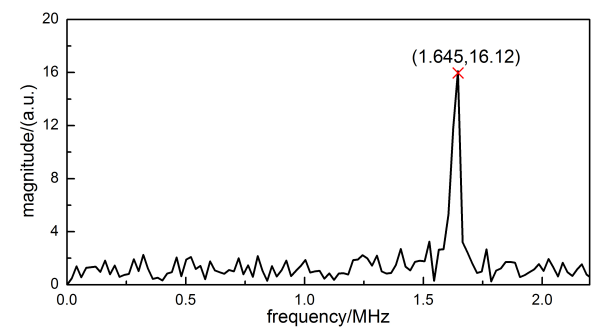

Fig. 11. Spectrum of turn-by-turn position.

\section{Conclusion}

A logarithm processing algorithm used to calculate beam transverse size and position based on SR light in HLS II is developed and preliminary experimental results are given. The measurement results are in good agreement with the simulation results after the channel inconsistency is calibrated. Vertical fractional tune of 0.3628 is obtained according to the turn-by-turn beam position 
which is very close to the nominal value 0.3621 . More experiments will be conducted in multiple bunches filling mode, and effective ways to excite the obvious change of beam size should be applied to study beam instability and beam dynamics.

\section{References}

1 Kube G. Review of Synchrotron Radiation Based Diagnostics for Transverse Profile Measurements. Proceedings of DIPAC 2007. Venice, Italy, 2007. 6-10

2 Bogomyagkov A V, Gurko V F, Zhuravlev A N et al. Rev. Sci. Instrum., 2007, 78: 043305
3 Palmer M, Dobbins J, Hartill D et al. Design and Implementation of an Electron and Positron Multibunch Turn-By-Turn Vertical Beam Profile Monitor in CESR. Proceedings of PAC 2007. Albuquerque, New Mexico, 2007. 4081-4083

4 TANG Kai, WANG Ji-Gang, SUN Bao-Gen et al. High Power Laser and Particle Beams, 2015, 27(7): 075101 (in Chinese)

5 Clarke J A. A Review of Optical Diagnostics Techniques for Beam Profile Measurements. Proceedings of EPAC 1994. Daresbury, UK, 1994. 1643-1645

6 CHENG Chao-Cai, XIAO Yun-Yun, SUN Bao-Gen et al. A New Method of Acquiring Fast Beam Transversal Profile in the Storage Ring. Proceedings of IPAC 2013. Shanghai, China, 2013. $556-558$ 